\title{
ASPECTOS RADIOLÓGICOS DA ATRESIA BRÔNOUICA: RELATO DE TRÊS CASOS E REVISÃO DA LITERATURA*
}

\author{
Alexandre da Silva Neu' ${ }^{1}$, Róger Eliandro Menezes ${ }^{1}$, Darcy de Oliveira llha² ${ }^{2}$ Antônio Carlos \\ Maciel $^{3}$, Rogério Fernandes Peixoto de Castro ${ }^{4}$
}

Resumo A atresia brônquica é uma anomalia congênita rara que usualmente produz massa justa-hilar com hipertransparência distal à radiografia simples. A maioria dos pacientes é jovem e não apresenta sintomas. A tomografia computadorizada confirma o diagnóstico, permitindo o manejo conservador nos casos assintomáticos. Os autores relatam três casos de atresia brônquica. Um caso, de particular interesse, estava associado a volumosa broncocele em paciente do sexo feminino, de $\mathbf{4 5}$ anos de idade, que apresentou quadro sintomático de infecção respiratória. $O$ diagnóstico foi estabelecido por meio dos achados da radiologia convencional, da tomografia computadorizada e da comparação com radiografias convencionais e tomografia linear prévios, que já demonstravam alterações. Realizou-se uma revisão sobre os achados clínicos e radiológicos dessa malformação.

Unitermos: Atresia brônquica; Broncocele; Mucocele brônquica.

Abstract Radiological aspects of bronchial atresia: report of three cases and review of the literature.

Bronchial atresia is a rare congenital abnormality that usually shows a juxta-hilar mass with distal radiolucency on conventional $x$-ray films. Most patients are young and have no symptoms. Computed tomography usually confirms the diagnosis, allowing conservative management of the asymptomatic cases. The authors report three cases of bronchial obstruction. One of the patients was of particular importance and interest, a 45-year-old female patient with symptoms of respiratory infection, due to the association with a bulky bronchocele. The diagnosis was made by analyzing the findings of conventional $x$-ray films and computed tomography, and the comparison with previous conventional x-ray films and linear tomography, which had already demonstrated abnormalities. A literature review on the clinical and radiological findings of this pulmonary malformation is presented.

Key words: Bronchial atresia; Bronchocele; Bronchial mucocele.

\section{INTRODUÇÃO}

A atresia brônquica congênita é uma malformação incomum do aparelho respiratório caracterizada pela obliteração focal da luz brônquica, mais comumente de um brônquio segmentar ${ }^{(\mathbf{1})}$. No brônquio atrésico, por não haver comunicação com a árvore central, há acúmulo de secreções, levando ao desenvolvimento da mucoce$\mathrm{le}^{(2)}$. Caracteristicamente, há hiperinsuflação segmentar ou lobar adjacente ${ }^{(3,4)}$, devido à ventilação pelos canais colaterais.

* Trabalho realizado no Serviço de Radiologia da Irmandade da Santa Casa de Misericórdia de Porto Alegre, Porto Alegre, RS.

1. Médicos Residentes de Radiologia da Irmandade da Santa Casa de Misericórdia de Porto Alegre.

2. Professor Titular da Cadeira de Radiologia da Universidade Federal do Rio Grande do Sul e da Fundação Faculdade Federal de Ciências Médicas de Porto Alegre.

3. Médico Chefe do Serviço de Radiologia da Irmandade da Santa Casa de Misericórdia de Porto Alegre.

4. Médico Radiologista do Serviço de Radiologia da Irmandade da Santa Casa de Misericórdia de Porto Alegre.

Endereço para correspondência: Dr. Alexandre da Silva Neu. Rua Duque de Caxias, 339, apto. 306, Centro. Porto Alegre, RS 90010-282. E-mail: neu@santacasa.tche.br

Recebido para publicação em 12/6/2002. Aceito, após revisão, em 7/10/2002.
Observa-se, ainda, redução da vascularização no segmento ou lobo envolvido.

A maioria dos pacientes é assintomática e os achados ao exame físico são mínimos. As características aos estudos de imagem são altamente sugestivas do diagnóstico. A tomografia computadorizada (TC) é o método de escolha para o diagnóstico.

\section{RELATO DOS CASOS}

\section{Caso 1}

Paciente de 45 anos de idade, sexo feminino, casada, branca, procurou o serviço médico por apresentar febre e falta de ar, sem outras queixas. Negava tabagismo ou uso de drogas. Relatou um episódio de pneumonia há 16 anos. Realizou-se radiografia de tórax, que demonstrou volumosa opacidade justa-hilar esquerda, acompanhada de redução da trama vascular pulmonar (Figura 1). Foram solicitados os exames anteriores e uma TC.

A TC evidenciou lesão expansiva de aproximadamente $12,0 \times 8,0 \times 6,0 \mathrm{~cm}$, de contornos lisos, precisos e lobulados, com densidade máxima de 50 unidades Hounsfield (UH), que não se impregnou pelo meio de contraste intravenoso (Figuras 2 e 3). Havia aprisionamento de ar com hiperinsuflação distal.

A análise retrospectiva da radiografia de tórax e da tomografia linear, realizadas em agosto de 1984, permitiu a identificação de brônquios segmentares e subsegmentares distendidos por muco, com aspecto digitiforme, que na ocasião foram considerados como vasos dilatados (Figuras 4 e 5).

A paciente foi submetida a segmentectomia ápico-posterior do lobo superior esquerdo. Macroscopicamente, foi identificada cavidade com paredes de 1,0 a 3,0 $\mathrm{mm}$ de espessura, parcialmente septada, com alguns pequenos recessos, provavelmente brônquios periféricos obstruídos.

\section{Caso 2}

Paciente de 18 anos de idade, sexo masculino, branco, solteiro, assintomático. Foi realizada radiografia de tórax na avaliação 


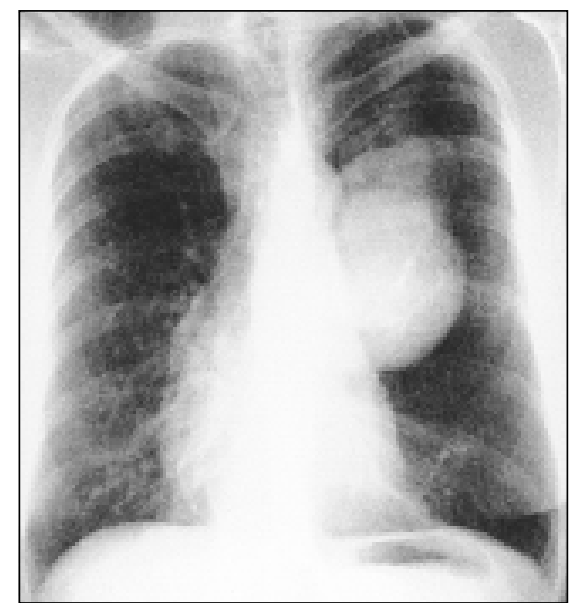

1

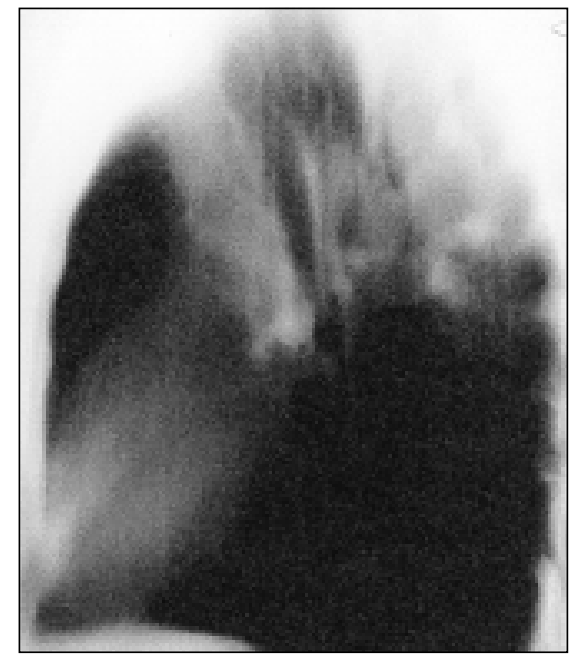

4

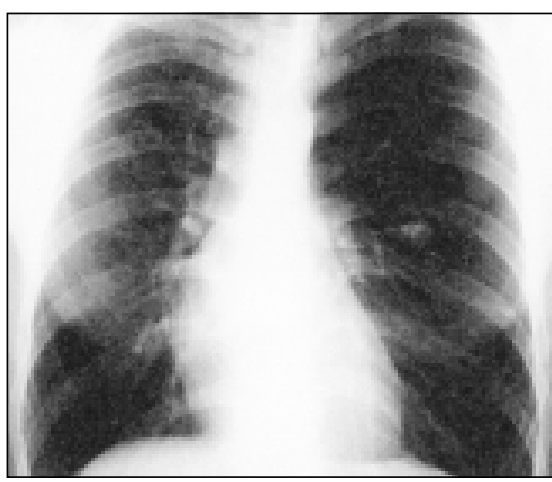

Figura 6. Caso 2. Pequena opacidade justa-hilar esquerda, associada a hipertransparência do lobo superior esquerdo.

pré-operatória de cirurgia corretora de estrabismo. Este exame demonstrou uma pequena opacidade justa-hilar esquerda com hipertransparência periférica associada (Figura 6).

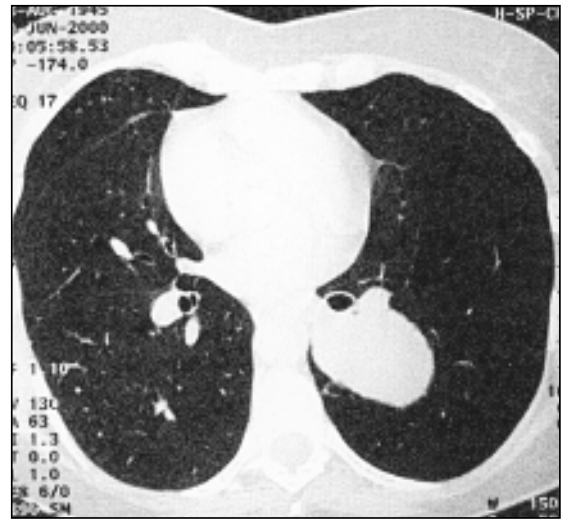

2

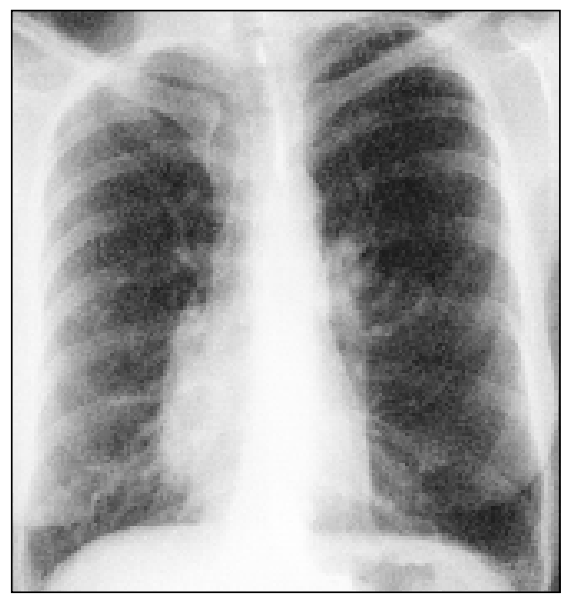

5

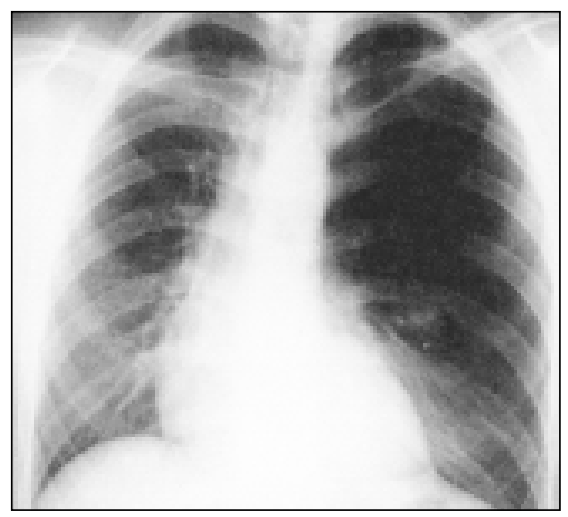

Figura 7. Caso 2. Radiografia em expiração mostrando aprisionamento de ar no lobo superior esquerdo.

Foi realizada radiografia de tórax em expiração, que revelou aprisionamento de ar no lobo superior esquerdo (Figura 7).

A TC de tórax confirmou o achado, demonstrando a mucocele do brônquio lobar

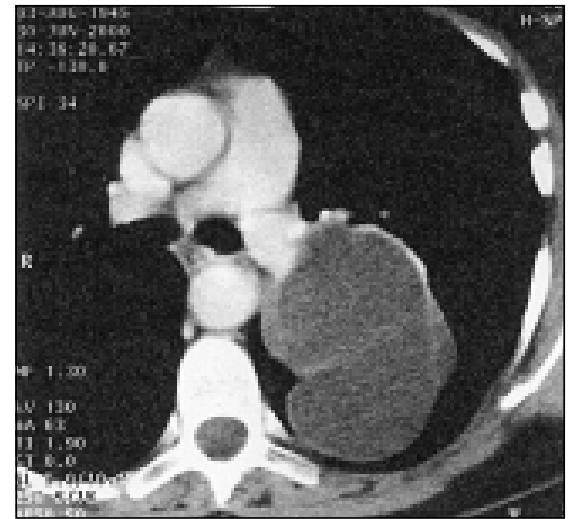

3

Figura 1. Caso 1. Radiografia frontal de tórax mostrando volumosa opacidade polilobulada justa-hilar esquerda, com rarefação das estruturas vasculares no lobo superior esquerdo.

Figura 2. Caso 1. Tomografia computadorizada de alta resolução de tórax mostrando massa justa-hilar esquerda, com rarefação das estruturas vasculares adjacentes e hipertransparência.

Figura 3. Caso 1. A lesão tumescente polilobulada não apresentou impregnação pelo contraste endovenoso.

Figura 4. Caso 1. Tomografia linear demonstrando opacidades justa-hilares em "dedos de luva".

Figura 5. Caso 1. Radiografia frontal de tórax, realizada há 16 anos, apresentando hipertransparência e hiperexpansão do lobo superior esquerdo.

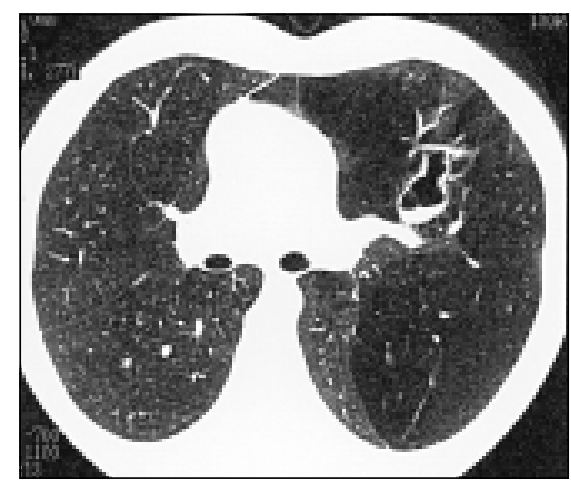

Figura 8. Caso 2. Broncocele com formação de nível hidroaéreo justa-hilar esquerdo, com hiperexpansão do parênquima pulmonar adjacente.

superior esquerdo como uma opacidade justa-hilar, que não sofreu realce ao contraste intravenoso, e o enfisema lobar periférico correspondente (Figura 8). O manejo foi conservador. 


\section{Caso 3}

Paciente de 28 anos de idade, sexo masculino, branco, solteiro, há um ano apresentando chiado no peito e dispnéia progressiva, atualmente aos médios esforços. Sem histórico de doenças respiratórias na infância e de tabagismo.

Ao exame físico apresentava marcado hipocratismo digital. A ausculta pulmonar revelou murmúrio vesicular uniformemente distribuído, sem ruídos adventícios. Paciente eupnéico.

A radiografia de tórax evidenciou opacidade nodular justa-hilar à esquerda, com aproximadamente $4,0 \mathrm{~cm}$ de diâmetro, acompanhada de hiperinsuflação no terço superior deste hemitórax (Figuras 9 e 10).

O paciente foi submetido a lobectomia superior esquerda, com a identificação de grande área cística, com paredes cinzentas e translúcidas, correspondendo a bolha de enfisema. Adjacente a esta, havia cavidade contendo material mucóide, que, quando removido, evidenciou brônquio dilatado com sua porção proximal atrésica. Havia continuidade com a via aérea distal.

\section{DISCUSSÃO}

Os primeiros relatos de atresia brônquica, na literatura, foram de Falor e Kyriakides ${ }^{(5)}$, em 1949. A impacção mucóide foi reconhecida inicialmente por Shaw, em $1951^{(6)}$, seguido por $\mathrm{Greer}^{(7)}$, em 1952. Em 1953, Ramsay ${ }^{(8)}$ e Ramsay e Byron ${ }^{(9)}$ descreveram uma série de pacientes com alterações císticas nos pulmões. Entretanto, apenas em 1963 Simon e Reid ${ }^{(\mathbf{1 0})}$ descreveram detalhadamente três casos de atresia brônquica segmentar e estabeleceram os critérios diagnósticos.

A representação tomográfica computadorizada dos achados ao estudo radiográfico simples de tórax foi realizada no trabalho de Cohen et al. ${ }^{(11)}$, em 1980.

\section{Embriologia}

A atresia brônquica é congênita, mas o momento preciso de sua ocorrência é discutível $^{(12-15)}$. O mecanismo da atresia não é conhecido.

$\mathrm{O}$ parênquima pulmonar origina-se como um broto dorsal do mesênquima entérico. A formação da árvore brônquica ocorre a partir da $4^{\mathrm{a}}$ semana de vida intra-

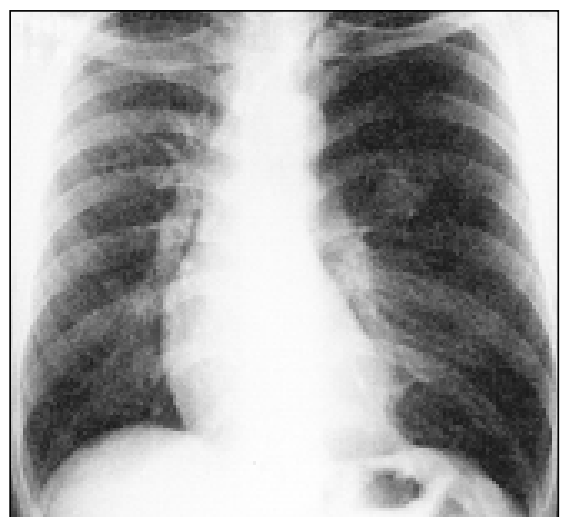

A

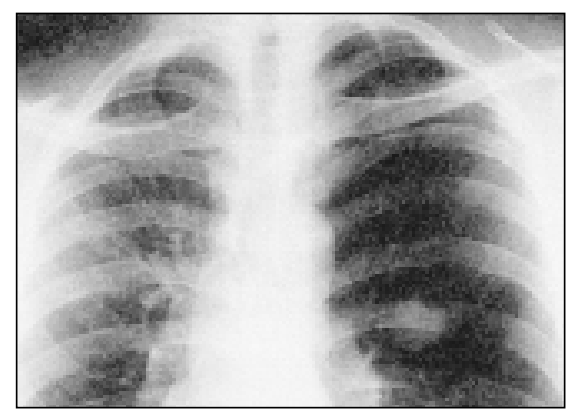

B

Figura 9. Caso 3. Opacidade justa-hilar esquerda, com hiperexpansão do lobo superior esquerdo.

uterina e se completa na $15^{\mathrm{a}}$ semana. Porém, ao nascimento, os alvéolos são rudimentares, sendo que a ventilação é responsável pelo aumento do número de alvéolos no adulto ${ }^{(16-18)}$

Em analogia ao que ocorre na atresia intestinal, uma interrupção no suprimento sanguíneo tem sido postulada como fator etiológico na atresia brônquica. Segundo Bucher e Reid e Curry e Curry, um insulto vascular isquêmico ocorrendo após a $15^{\mathbf{a}}$ semana de vida intra-uterina explicaria a obliteração focal do brônquio, permanecendo a árvore brônquica distal normal e os alvéolos hipoplásicos ${ }^{(7,9,13,14,19)}$. Evidências que apóiam essa hipótese incluem a alta frequiência de envolvimento do brônquio lobar superior esquerdo, uma área de reconhecida instabilidade no desenvolvimento embrionário tardio. Isto, entretanto, não explica os casos em que não há vestígios de conexão no segmento atrésico e a presença de anomalias associadas, como os cistos broncogênicos, que se desenvolvem precocemente, quando surgem os brotos brônquicos, sendo proposta uma lesão isquêmica entre a $5^{\mathrm{a}}$ e a $15^{\mathrm{a}}$ semanas de vida intra-uterina ${ }^{(\mathbf{1 1 , 2 0 , 2 1 )}}$. Uma vez que o sítio da atresia pode apresentar vários graus de restos vestigiais, é razoável supor que o insulto vascular possa ocorrer em diferentes momentos, entre a $5 \underline{a}$ semana de gestação até o desenvolvimento da árvore brônquica estar completo.

\section{Fisiopatologia}

O parênquima suprido pelo brônquio atrésico não se torna atelectásico, mas enfisematoso ${ }^{(22)}$. $\mathrm{O}$ ar atinge o parênquima através dos canais colaterais de ventilação intra-alveolares (poros de Kohn), broncoalveolares (canais de Lambert) e interbronquiolares (Martin) ${ }^{(2,23-27)}$. Esses mecanismos ocorrem entre os segmentos, mas não entre os lobos. A ventilação colateral é menos efetiva na expiração do que na inspiração, levando a um alçaponamento significativo de $\operatorname{ar}^{(28)}$.

O brônquio distal à obstrução continua a secretar muco das glândulas submucosas e das células "globet". Esse muco fica coletado distalmente à obstrução, levando à dilatação progressiva da mucocele brônquica. O grau de dilatação depende da produção mucóide e da organização da cartilagem da parede do brônquio ${ }^{(9,29,30)}$.

\section{Achados patológicos}

O diagnóstico patológico da atresia brônquica congênita depende de uma análise cuidadosa do pulmão ressecado. A atresia, na maioria das vezes, é segmentar. O segmento ou lobo envolvido é marcadamente enfisematoso e não apresenta pigmento antracósico, já que esta parte do parênquima nunca foi diretamente ventila-

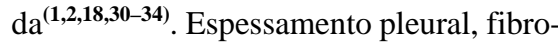
se e circulação colateral são resultado de infecções recorrentes. Não há conexão com a árvore brônquica central, apesar de poder estar presente remanescente de tecido fibroso, septo ou membrana ${ }^{(\mathbf{8 , 3 5})}$. O brônquio proximal termina em fundo de saco e as ramificações estão dilatadas.

A mucocele brônquica é delineada por epitélio ciliado, cuboidal ou colunar, dependendo do grau de distensão mucosa. Imaturidade histológica e anormalidades 
na cartilagem brônquica podem estar presentes $^{(\mathbf{3 1}, \mathbf{3 6})}$.

\section{Diagnóstico}

A atresia brônquica é uma malformação congênita incomum que envolve quase invariavelmente um único segmento brônquico. A maioria dos pacientes é jovem no momento do diagnóstico, mas a faixa etária dos casos descritos na literatura variou de 2 dias a 41 anos. Não há predominância de sexo ${ }^{(\mathbf{1 1 , 2 1 , 2 2 , 2 4 , 3 0 )}}$. Mais de dois terços dos pacientes são assintomáticos ${ }^{(\mathbf{3 0 , 3 1})}$ no momento da apresentação. Porém, sintomas de infecção, dispnéia e tosse têm sido relatados ${ }^{(\mathbf{2 4 , 3 1})}$. Ao exame físico, há redução localizada do murmúrio vesicular na área afetada. Jederlinic et al. ${ }^{(\mathbf{3 1})}$ descreveram um caso com deformidade associada da caixa torácica e outro com hipoplasia ipsilateral dos músculos peitorais.

Várias anormalidades têm sido descritas em associação com a atresia brônquica, incluindo seqüestro pulmonar, malformação adenomatóide congênita, enfisema lobar congênito, cistos broncogênicos, defeitos pericárdicos, retorno venoso pulmonar anômalo e pulmões aplásicos ${ }^{(\mathbf{6})}$.

O segmento ápico-posterior do lobo superior esquerdo é o mais freqüentemente acometido $^{(\mathbf{1}, 3,10,11,14,15,22,24,25,31,37)}$. A alta incidência de variações anatômicas nos brônquios do lobo superior esquerdo sugere um alto grau de instabilidade embriológica, o que explicaria essa predileção anatômica.

A fisiopatologia da atresia brônquica leva a achados radiológicos característicos. À radiografia simples de tórax, a broncocele é tipicamente identificada como uma opacidade arredondada ou fusiforme, ramificada, que irradia a partir do hilo pulmonar, descrita como dedos de luva, cachos de uva, forma de V ou Y. Ocasionalmente, há nível líquido ${ }^{(1,11,22,24,31,38)}$. A hipertransparência periférica associada representa a hiperinsuflação do parênquima envolvido e a correspondente oligoemia. Variações ocorrem e, ocasionalmente, não é identificada a opacidade ${ }^{(30)}$. Em pacientes mais velhos, a broncocele terá aumentado o suficiente, tornando a lesão típica.

A TC é o método de escolha para o diagnóstico de atresia brônquica ${ }^{(\mathbf{1}, 11,24,32)}$, sendo muito sensível na demonstração dos achados típicos de alçaponamento de ar, que pode claramente ser identificado em expiração ${ }^{(1,11,37)}$. A TC também é útil para excluir a presença de massas hilares e demonstrar a broncocele, que não se impregna pelo meio de contraste. Adicionalmente, a TC de alta resolução permite delinear e localizar com maior precisão a broncocele, em virtude da melhor resolução espacial e da constante identificação das cissuras $^{(39)}$. A TC espiral com reconstrução multiplanar é útil na distinção entre a broncocele e outras lesões nodulares, incluindo malformações vasculares ${ }^{(40)}$.

Os coeficientes de atenuação não são úteis para predizer o conteúdo dos brônquios dilatados, apesar de a baixa densidade $(-5$ a $+20 \mathrm{UH})$ sugerir conteúdo mucóide. Entretanto, números de 30 a 50 UH foram encontrados, devidos a secreções espessadas ou tumorações ${ }^{(\mathbf{4 0 )}}$.

A TC com o uso de meio de contraste endovenoso exclui anormalidades vasculares, tornando desnecessário o emprego de angiografia $^{(31,40)}$.

A broncografia e a broncoscopia têmse mostrado incômodas, dispendiosas e pouco elucidativas ${ }^{(\mathbf{1 1 , 3 0 , 3 1 , 4 0 )} \text {. }}$

A ressonância magnética demonstra as mucoceles brônquicas hiperintensas nas ponderações em T1 e T2 $2^{(4)}$, mas não consegue definir a hipertransparência distal que é visível na TC.

As broncoceles podem ser facilmente diferenciadas das malformações vasculares e de causas adquiridas de obstrução brônquica $^{(41)}$. Nos casos em que a TC espiral deixa dúvidas a respeito da perviedade do brônquio central, a broncoscopia deve ser considerada $^{(31)}$.

Considerados no diagnóstico diferencial, os cistos pulmonares broncogênicos e o sequiestro intralobar raramente exibem hiperinsuflação ${ }^{(3,4,30,31,40,42,43)}$.

\section{Tratamento}

O tratamento cirúrgico há muito tempo não é necessário para o diagnóstico e, em pacientes assintomáticos, o risco do procedimento supera os possíveis benefícios ${ }^{(\mathbf{3 0})}$.

A segmentectomia ou a lobectomia ficam reservadas aos casos em que há infecções de repetição e comprometimento por compressão de estrutura parenquimatosa pulmonar adjacente ${ }^{(4,11,40)}$.

\section{CONCLUSÕES}

O achado tomográfico computadorizado de impacção mucóide com enfisema subjacente, eventualmente seguido por broncoscopia para demonstrar a perviedade do brônquio central, é virtualmente patognomônico de broncomucocele secundária a atresia brônquica congênita. Nos casos típicos, não há necessidade de investigações adicionais.

Em pacientes assintomáticos, o manejo conservador é atualmente indicado.

\section{REFERÊNCIAS}

1. Ward S, Morcos SK. Congenital bronchial atresia - presentation of three cases and a pictorial review. Clin Radiol 1999;54:144-8.

2. Williams AJ, Schuster SR. Bronchial atresia associated with a bronchogenic cyst. Evidence of early appearance of atretic segments. Chest 1985;87: 396-8.

3. Felson B. Mucoid impaction (inspissated secretions) in segmental bronchial obstruction. Radiology 1979;133:9-16.

4. Ko SF, Lee TY, Kao CL, et al. Bronchial atresia associated with epibronchial right pulmonary artery and aberrant right middle lobe artery. Br J Radiol 1998;71:217-20.

5. Falor WH, Kyriakides AH. Ectopic bronchi. J Thorac Cardiovasc Surg 1949;18:252-60.

6. Shaw RR. Mucoid impaction of the bronchi. J Thorac Surg 1951;22:149-63.

7. Greer AE. Mucoid impaction of the bronchi. Ann Intern Med 1957;46:506-22.

8. Ramsay BH. Mucocele of the lung due to congenital obstruction of segmental bronchus. Dis Chest 1953;24:96-103.

9. Ramsay BH, Byron FX. Mucocele, congenital bronchiectasis and bronchogenic cysts. J Thorac Surg 1953;26:21-30.

10. Simon G, Reid L. Atresia of an apical bronchus of the left upper lobe - report of three cases. Br J Dis Chest 1963;57:126-32.

11. Cohen AM, Solomon EH, Alfidi RJ. Computed tomography in bronchial atresia. AJR 1980;135: 1097-9.

12. Boyden EA. A study of the patterns of the segmental bronchi and related pulmonary vessels. New York: MCGraw-Hill, 1955.

13. Bucher U, Reid L. Development of intrasegmental bronchial tree: the pattern of branching and development of cartilage at various stages of intrauterine life. Thorax 1961;16:207.

14. Curry TS III, Curry GC. Atresia of the bronchus to the apical posterior segment of the left upper lobe. Am J Roentgenol Radium Ther Nucl Med 1966;98: 350-3.

15. Waddell JA, Simon G, Reid L. Bronchial atresia of the left upper lobe. Thorax 1965;20:214-8.

16. Remy-Jardin M, Remy J, Ribert M. Atrésie bronchique: critères diagnostiques et considerations embryologiques. Diagn Interv Radiol 1989;1:45-51.

17. Hislop A, Reid L. In: Davis JA, Dobbing J, eds Scientific foundations of paediatrics. London: Heinemann Medical Books, 1982. 
18. Généreux GP. Bronchial atresia: a rare cause of unilateral lung hypertranslucency. J Can Assoc Radiol 1971;22:71-82.

19. Louw JH, Barnard CN. Congenital intestinal atresia: observations on its origin. Lancet 1955;2:1065.

20. Spencer H. Pathology of the lung. New York: Macmillan, 1962.

21. Williams LE, Murray GF, Wilcox BR. Congenital atresia of the bronchus. J Thorac Cardiovasc Surg 1974;68:957-61.

22. Wilson EB, Jones RJ. Segmental bronchial atresia of the left upper lobe with resultant bronchial mucocele. J Thorac Cardiovasc Surg 1972;63:48690

23. Lacquet LK, Fornhoff M, Dierickx R, Buyssens N Bronchial atresia with corresponding segmental pulmonary emphysema. Thorax 1971;26:68-73.

24. Ouzidane L, Benjelloun A, el Hajjam M, Ksiyer M, Abid A. Segmental bronchial atresia - a case repor and a literature review. Eur J Pediatr Surg 1999;9: 49-52.

25. Oh KS, Dorst JP, White JJ, Haller JA Jr, Johnson BA, Byme WD. The syndrome of bronchial atresia or stenosis with mucocele and focal hyperinflation of the lung. Johns Hopkins Med J 1976;138:48-53.

26. Avery ME, Fletcher BD. The lung and its disorders in the newborn infant. Philadelphia: WB Saunders, 1974.

27. Lambert MW. Acessory bronchiole-alveolar communications. J Pathol Bacteriol 1955;70:311-4.
28. Haller JA Jr, Tepas JJ III, White JJ, Pickard LR, Robotham JL. The natural history of bronchial atresia. Serial observations of a case from birth to operative correction. J Thorac Cardiovasc Surg 1980; 79:868-72.

29. Talner LB, Gmelich JT, Liebow AA, Greenspan RH. The syndrome of bronchial mucocele and regional hyperinflation of the lung. Am J Roentgenol Radium Ther Nucl Med 1970;110:675-86.

30. Meng RL, Jensik RJ, Faber LP, Matthew GR, Kittle CF. Bronchial atresia. Ann Thorac Surg 1978;25: 184-92.

31. Jederlinic JP, Sicilian LS, Baigelman W, Gaensler EA. Congenital bronchial atresia. A report of four cases and a review of the literature. Medicine (Baltimore) 1987;66:73-83.

32. Coelho BCD, Skaf AY, Maciel Jr FS, Bergamaschi $\mathrm{CV}$, Faria GS, Vega HO. Atresia brônquica: relato de um caso e revisão da literatura. Radiol Bras 1995;28:339-41.

33. Fusonie D, Molnar W. Anomalous pulmonary venous return, pulmonary sequestration, bronchial atresia, aplastic right upper lobe, pericardial defect and intrathoracic kidney. An unusual complex of congenital anomalies in one patient. Am J Roentgenol Radium Ther Nucl Med 1966;97:350-4

34. Hanna EA, Vattanapat S, Derrick JR. Congenital lobar emphysema with bronchial atresia. Ann Thorac Surg 1969;7:357-61.

35. Culiner MM. Collateral ventilation and the hyper- lucent lung. Am J Med 1964;36:395-403.

36. Tsuji S, Heki S, Kobara Y, Sato A. The syndrome of bronchial mucocele and regional hyperinflation of the lung. Report of four cases. Chest 1973;64: 444-7.

37. Warner JO, Rubin S, Heard BE. Congenital lobar emphysema: a case with bronchial atresia and abnormal bronchial cartilages. Br J Dis Chest 1982; 76:177-84.

38. Carlson V, Martin JE, Keegan JM, Dailey JE. Roentgenographic features of mucoid impaction of the bronchi. Am J Roentgenol Radium Ther Nucl Med 1966;96:947-52.

39. Remy-Jardin M, Remy J, Ribert M, Gosselin B. Bronchial atresia: diagnostic criteria and embryologic considerations. Rev Im Med 1989;1:53-9.

40. Pugatch RD, Gale ME. Obscure pulmonary masses: bronchial impaction revealed by CT. AJR 1983; 141:909-14.

41. Beigelman C, Howarth NR, Chartrand-Lefebvre C Grenier P. Congenital anomalies of tracheobronchial branching patterns: spiral CT aspects in adults. Eur Radiol 1998;8:79-85

42. Finck S, Milne ENC. A case report of segmental bronchial atresia: radiologic evaluation including computed tomography and magnetic resonance imaging. J Thorac Imaging 1988;3:53-7.

43. Rossoff LJ, Steinberg H. Bronchial atresia and mucocele: a report of two cases. Respir Med 1994 88:798-91. 